\title{
Un análisis experimental sobre la elección del medio de transporte*
}

\author{
Alessandro Innocenti \\ Universidad de Siena \\ Patrizia Lattarulo \\ IRPET (Instituto Regional para la \\ Planeación Económica de la Toscana) \\ Maria Grazia Pazienza \\ Universidad de Florencia
}

\begin{abstract}
Resumen
Este artículo extiende trabajos previos sobre elección de "ruta" analizando la elección de medio de transporte. Estudiamos dos entornos. En el primero, los sujetos eligen si trasladarse en metro o en coche. Los costes de metro son fijos, mientras los costes de trasladarse en coche son inciertos y dependen del efecto conjunto de eventos aleatorios y de la congestión de tráfico. En el segundo, los sujetos pueden viajar en coche o en autobús, cuyos costes dependen en ambos casos de factores aleatorios y de la congestión. Identificamos una fuerte preferencia por el automóvil y una tendencia a no cambiar el medio previamente elegido, de manera que se confirma la primera elección y se actualizan imperfectamente las expectativas pre-existentes sobre los tiempos de viaje.
\end{abstract}

Palabras clave: elección del medio de transporte, procesamiento de la información, aprendizaje, expectativas, sesgos cognitivos.

Códigos JEL: C91, D83, R41, R48.

\section{Abstract}

This study extends previous experimental work on route choice by analyzing the case of travel mode choice. Two different settings are considered. In the first setting, subjects have to decide if commute by car or by metro. Metro costs are fixed, while car costs are uncertain and determined by the joint effect of casual events and traffic congestion. In the second, subjects can travel by car or by bus, whose costs are both determined by the combination of chance and congestion. We find that subjects exhibit a marked preference for cars, are inclined to travel mode stickiness and, more specifically, to confirm their first choice, and update imperfectly their expectations on travel times.

Keywords: travel mode choice, information processing, learning, expectations, cognitive biases.

JEL codes: C91, D83, R41, R48.

* Esta investigación forma parte de un proyecto de trabajo de IRPET (Instituto Regional para la Planeación Económica de la Toscana). Agradecemos a Roberto Ricciuti por sus valiosos comentarios, a Maria Luisa Maitino por el análisis estadístico y a Francesco Lo Magistro por la asistencia en el laboratorio. Traducción a cargo de Pablo Brassiolo. 


\section{Introducción}

Este artículo presenta un análisis experimental de la elección de medio de transporte cuando están disponibles dos medios de transporte. El enfoque convencional sobre este tema asume que los viajeros toman decisiones maximizando la utilidad individual y, consecuentemente, minimizando los tiempos de viaje (Mirchandani y Soroush, 1987; Cascetta, 1989; Friesz y otros, 1994). Esta hipótesis implica que los individuos tienen en cuenta correctamente toda la información disponible y son perfectamente racionales. Por el contrario, las teorías no convencionales de toma de decisión bajo incertidumbre se desvían de las definiciones estrictas de racionalidad, especialmente cuando el tomador de decisiones se enfrenta a elecciones repetidas que involucran pagos inciertos (Starmer 2000). En estos entornos, los modelos de comportamiento introducen una gran variedad de supuestos sobre los procesos de aprendizaje y adquisición de información, basados en resultados tanto de campo como de laboratorio. En particular, los estudios psicológicos y cognitivos señalan que los tomadores de decisiones tienen racionalidad limitada en la actividad de procesamiento de la información. Para investigar las implicaciones de esta teoría en el laboratorio, diseñamos un modelo de elección de viaje como un proceso repetido en el que los viajeros actualizan sus expectativas acerca de los tiempos de viaje sobre la base de su propia experiencia y de la información pública.

Nuestro diseño experimental incluye dos entornos. En el primero, los sujetos eligen si trasladarse en metro o coche. Los costes del metro son fijos, mientras que los costes de trasladarse en coche son inciertos y dependen del efecto conjunto de eventos aleatorios y la congestión de tráfico. En el segundo de los entornos estudiados, los sujetos pueden viajar en coche o en autobús, cuyos costes dependen en ambos casos de factores aleatorios y de la congestión de la ruta. La utilidad de los viajeros depende solamente de los tiempos de viaje, los cuales se convierten a costes monetarios. Los sujetos reciben información sobre los tiempos reales de viaje en los dos medios disponibles para poder analizar cómo forman y actualizan sus expectativas sobre los tiempos de viaje.

Este artículo se divide en cinco secciones. En la Sección 2 se resume la investigación del comportamiento en elección de ruta y medio de transporte. La Sección 3 describe el diseño experimental. La Sección 4 discute los resultados y algunas implicaciones. Finalmente, las conclusiones se presentan en la Sección 5.

\section{Análisis de la literatura}

La investigación del comportamiento sobre los determinantes de la elección del medio de transporte se centra en tres temas principales. El primero está relacionado con los efectos de la experiencia y la información en los procesos de aprendizaje, el segundo analiza el impacto de los hábitos de las decisiones de los viajeros y el tercero aborda la relevancia de su actitud frente al riesgo. 
La literatura sobre la elección del medio de transporte se basa en estudios de la elección de ruta, los cuales son objeto de una gran cantidad de evidencia empírica y experimental. Un objetivo común del análisis es el tema de la coordinación de los viajeros. Tanto el medio de transporte como la elección de ruta pueden ser representados como juegos de coordinación en los cuales el pago que cada viajero obtiene es condicional a su capacidad de divergir de o de converger a las elecciones de los otros viajeros. En estos juegos, los jugadores racionales tienen que coordinar sobre un equilibrio. En el caso de la elección del medio de transporte, los viajeros tienen que optar entre medios alternativos de forma que sus utilidades individuales se igualen. Este proceso depende estrictamente de las expectativas sobre las elecciones de los otros viajeros, las cuales se forman sobre la base de experiencia previa e información pública. La evidencia tanto de campo como de laboratorio respalda la noción de que estos procesos de aprendizaje están distorsionados por limitaciones cognitivas, lo cual debilita la validez de la hipótesis de racionalidad.

En el contexto de la elección de ruta, Avineri y Prashker (2006) muestran en el laboratorio que facilitar información más precisa a los viajeros sobre los tiempos reales de viaje no necesariamente incrementa su propensión a minimizar los costes de viaje. Sus resultados pueden interpretarse como evidencia de que el procesamiento de información está afectado por limitaciones en procesos cognitivos como aprendizaje, memoria y resolución de problemas. Una primera fuente de distorsión está dada por el hecho de que los tiempos esperados de viaje se infieren por tiempos de viajes experimentados. Dado que los datos de muestra son estimadores sesgados de la distribución poblacional, la percepción subjetiva de los viajeros puede ser muy diferente de los valores reales (Kahneman y Tversky, 1973; Kareev y otros, 2002). En general, si la variabilidad de los tiempos de viaje aumenta, tal como lo postula el diseño experimental de Avineri y Prashker (2006), la distorsión se hace más pronunciada. En segundo lugar, la racionalidad de los viajeros puede quedar afectada por una memoria restringida. Kareev y otros (1997) muestran que las diferencias individuales en la capacidad de memoria implican que los tomadores de decisiones procesan muestras de tamaños distintos y mantienen expectativas subjetivas diferentes. La investigación empírica (Srinivasan y Mahamassani, 1999; Abdel-Aty y Abdallah; 2004) muestra que la información sobre calendarios o demoras de viaje se suele procesar de manera imprecisa. Haciendo hincapié en algunos tipos de mensajes más que en otros, la información en tiempo real se recolecta y procesa en forma secuencial y no instantánea o exhaustiva, y las decisiones de los viajeros se toman de forma aproximada o heurística, incluyendo límites de tolerancia y rutinas. En otro experimento, Ben-Elia y otros (2008) testean los efectos conjuntos de la experiencia personal y la información en tiempo real en las decisiones de los viajeros y concluyen que entre ambas existe substitución, más que complementariedad. Su principal resultado es que la información se procesa mejor cuando los viajeros carecen de experiencia a largo plazo con relación a la distribución de los tiempos de viaje. Estos resultados tienen implicaciones negativas para las políticas de transporte, que crecientemente se apoyan en el hecho de que los viajeros cuentan con infor- 
mación en tiempo real sobre los viajes, la cual es más comprehensiva y precisa que la información basada solamente en la experiencia personal ${ }^{1}$.

En efecto, el hecho de que los medios de transporte sean habitualmente elecciones repetidas afecta negativamente la manera en la que deciden incluso sujetos racionales (Gärling, 1998). En este contexto, los hábitos desatan reacciones automáticas a los factores externos, que son tomados sin mucha deliberación (Aarts y otros, 1997; Bamberg y otros, 2003). Una reseña elaborada por Mahmassani (1996) sobre el comportamiento de viajeros repetitivos señala que son guiados principalmente por un razonamiento heurístico. Mahmassani y Liu (1998) proponen una lista de estrategias de comportamiento seguidas por viajeros en cambios de decisiones y las asocian con las características de la información sobre demoras y salidas. Otros estudios empíricos (Verplanken y otros, 1994; Verplanken y Aarts, 1999) corroboran este punto de vista mostrando que si el comportamiento habitual se fortalece, los esfuerzos mental y cognitivo se reducen a un mínimo y la información adicional es menos analizada. Una investigación de laboratorio de (Avineri y Prashker, 2003) brinda evidencia de que la repetición de elecciones genera el llamado efecto de variabilidad de pagos, de acuerdo con el cual el incremento en la variabilidad de los tiempos de viaje hace las decisiones más heterogéneas y reduce la tasa de maximización. Ben-Elia y otros (2008) corroboran este resultado al observar que tomar decisiones sin una descripción de las distribuciones relevantes incrementa el grado de aleatoriedad.

Otro tema clave en la investigación sobre decisiones de viajeros es la actitud frente al riesgo. La investigación empírica apoya la hipótesis de que los viajeros habitualmente son aversos al riesgo (Abdel-Aty y Abdallah, 2004). Este supuesto se contradice con los resultados de laboratorio de Katsikopoulos y otros (2000) para la elección de ruta, quienes emplean un simulador de conducción para investigar el comportamiento de los conductores. En este artículo, los sujetos eligen entre una ruta de referencia, cuyo tiempo de viaje es cierto, y algunas rutas alternativas, las cuales tienen un rango de tiempos asociados. Se comparan dos entornos. En el primero, que se refiere al dominio de las ganancias, la ruta alternativa tiene un tiempo de viaje esperado más corto que la ruta de referencia, mientras que en el segundo entorno, referido al dominio de las pérdidas, el tiempo esperado de viaje de la ruta alternativa es mayor que el de referencia. Los sujetos exhiben aversión por el riesgo para las ganancias y propensión al riesgo para las pérdidas. La evidencia que apoya esta teoría proviene de Ben-Elia y otros (2008), quienes muestran que una mejor información aumenta el riesgo de un comportamiento buscador de riesgo, reduce la exploración inicial e incrementa la heterogeneidad de las elecciones. Finalmente, Scheiner y Holz-Rau (2007) ofrecen argumentos empíricos y teóricos que sugieren que la elección del medio de transporte depende tanto de factores obje-

${ }^{1}$ Esta evolución es debida al creciente uso de Sistemas Avanzados de Información de Viajeros (ATIS, por sus siglas en inglés) tales como la transmisión de condiciones de tráfico, los Signos de Mensajes Variables (VMS, por sus siglas en inglés) y los sistemas de información celular. 
tivos como subjetivos. De acuerdo con su visión, los estilos de vida individuales juegan un rol importante en la determinación de los medios de transporte. Su argumento implica que la rigidez en el medio de transporte debe ser considerada un problema clave en el diseño de políticas de transporte efectivas.

\section{Diseño experimental}

Nuestros experimentos fueron llevados a cabo en la primavera de 2008 en la Universidad de Florencia, Italia. Enviamos la elección del medio de transporte a 62 sujetos (31 mujeres y 31 hombres). Los participantes eran estudiantes de pre-grado de las Facultades de Economía y Ciencias Políticas, reclutados de clases introductorias de economía y sin ninguna experiencia en este entorno de toma de decisiones.

Los experimentos fueron computarizados empleando una versión modificada del programa informático Z-tree (Fischbacher, 2007). Los sujetos fueron ubicados frente a los ordenadores en asientos separados para prevenir la comunicación directa o el contacto visual entre ellos. Además, se les aclaró que para los demás participantes no era posible atribuir las elecciones individuales a personas individuales. A cada sujeto se le otorgó un premio de 5 euros por participar. Al comienzo de cada sesión, los sujetos recibieron una dotación de 150 unidades experimentales. Al final de la sesión, a los sujetos se les pagó en efectivo de acuerdo a las unidades que tenían. Las unidades experimentales se convirtieron a euros a una tasa predeterminada conocida por los participantes antes del experimento. Cada sesión duró aproximadamente una hora y las ganancias promedio fueron de 18,40 euros, incluyendo el premio por participar.

Los sujetos recibieron instrucciones escritas, que además fueron leídas en voz alta por el experimentalista. Antes del comienzo el experimento, los participantes tuvieron la oportunidad de familiarizarse con el diseño en tres rondas de prueba no pagadas. Antes y después de esas rondas, los sujetos podían preguntar en voz alta cuestiones referidas a los procedimientos del experimento.

Realizamos tres tratamientos con diferentes grupos de personas. El diseño entre sujetos fue pensado para evitar efectos de arrastre de un tratamiento a otro. En el primer tratamiento, los sujetos tenían que elegir entre coche y metro, mientras que en el segundo tratamiento tenían que optar entre coche y autobús. El segundo y tercer tratamiento se diferenciaron únicamente por el coste fijo del autobús, que cambiaba de 1,0 a 0,8 para aislar, ceteris paribus, el efecto de una reducción en el precio del autobús.

La Tabla 1 resume nuestro diseño experimental mostrando el número y género de los participantes para cada tratamiento y sesión. 
TABLA 1

NÚMERO Y GÉNERO DE LOS PARTICIPANTES POR SESIÓN

\begin{tabular}{|c|c|c|}
\hline Sesión & Tratamiento & $\begin{array}{c}\text { Participantes } \\
\text { (femeninos + masculinos) }\end{array}$ \\
\hline 1 & Metro vs. Coche & $15(8+7)$ \\
2 & Metro vs. Coche & $15(7+8)$ \\
3 & Autobús 1,0 vs. Coche & $15(7+8)$ \\
4 & Autobús 0,8 vs. Coche & $17(9+8)$ \\
\hline Total & & $\mathbf{6 2}(\mathbf{3 1}+\mathbf{3 1})$ \\
\hline
\end{tabular}

En cada sesión, los sujetos tenían que elegir el medio de transporte durante 50 períodos. En cada período, tenían que seleccionar uno de los dos medios de transporte disponibles. Para cada medio de transporte, los sujetos estaban informados de los costes y de los tiempos de viaje esperados o programados. También se dejó claro que cualquier desviación del tiempo de viaje programado estaba asociada a un coste o ganancia monetaria.

Antes de elegir, los sujetos recibieron la siguiente información:

a) El tiempo esperado de viaje para cada medio de transporte;

b) Que el tiempo de viaje esperado en metro era fijo, mientras que el coche y el autobús tenían tiempos de viaje inciertos;

c) El tiempo de viaje en coche o autobús dependía de la congestión en el tráfico que a su vez dependía de las elecciones del medio de transporte de los demás sujetos, y de algunos factores impredecibles (clima, accidentes de tráfico, obras en rutas), cuyo efecto en el tiempo de viaje era seleccionado al azar por el ordenador antes de cada período;

d) El coste fijo de cada medio de transporte;

e) La penalización a pagar o el premio a recibir (0,5 unidades), por cada cinco minutos de retraso o por cada cinco minutos ganados sobre el tiempo esperado o programado de viaje, respectivamente.

Los parámetros del experimento fueron dados a conocer a los sujetos como se muestra en la Tabla 2. 
TABLA 2

PARÁMETROS DEL EXPERIMENTO DADOS A CONOCER A LOS PARTICIPANTES*

\begin{tabular}{|l|c|c|c|c|}
\hline Tratamiento & $\begin{array}{c}\text { Tiempo esperado } \\
\text { de viaje en coche } \\
\text { (en minutos) }\end{array}$ & $\begin{array}{c}\text { Coste } \\
\text { fijo del } \\
\text { coche }\end{array}$ & $\begin{array}{c}\text { Tiempo esperado } \\
\text { de viaje metro/ } \\
\text { autobús }\end{array}$ & $\begin{array}{c}\text { Coste fijo } \\
\text { metro/ } \\
\text { autobús }\end{array}$ \\
\hline Metro vs. coche & 25 & 1,5 & 30 & 1,0 \\
Autobús 1.0 vs. coche & 27 & 1,5 & 32 & 1,0 \\
Autobús 0.8 vs. coche & 27 & 1,5 & 32 & 0,8 \\
\hline
\end{tabular}

* Los tiempos y costes de viaje en metro eran fijos, mientras que los de autobús y coche eran variables.

En cada período, después de haber realizado la elección, cada participante recibía información privada sobre:

a) El tiempo de viaje real (ex post) de los dos medios disponibles;

b) El nivel de congestión de tráfico definido como moderado, intenso o caótico, con relación al porcentaje de participantes que elegían cada uno de los medios.

c) El coste monetario individual pagado por el viaje (incluyendo penalizaciones o recompensas adicionales) y el número restante de unidades.

Para examinar cómo se formaban y actualizaban las expectativas sobre los tiempos de viaje, los parámetros de la función de la cual el ordenador selecciona los tiempos (determinando parte de la desviación de los tiempos reales sobre los esperados) se mantuvieron desconocidos para los sujetos.

En el tratamiento metro, el componente casual del tiempo de viaje real en coche fue obtenido como una realización aleatoria de la distribución de probabilidad que se muestra en la Tabla 3.

TABLA 3

IMPACTO DE FACTORES CASUALES SOBRE EL TIEMPO DE VIAJE EN COCHE - TRATAMIENTO METRO

\begin{tabular}{|l|c|c|c|c|c|}
\hline & \multicolumn{5}{|c|}{ Tiempo de viaje en coche } \\
\hline $\begin{array}{c}\text { Tiempo de viaje } \\
\text { (en minutos) } \\
\text { Probabilidad }\end{array}$ & 20 & 25 & 30 & 35 & 40 \\
\hline
\end{tabular}

El valor esperado de esta distribución es veinticinco minutos, que era el tiempo de viaje esperado en coche dado a conocer a los sujetos. El tiempo de viaje real en coche dependía además de la congestión en el tráfico (Tabla 4). 
TABLA 4

IMPACTO DE LA CONGESTIÓN DE TRÁFICO EN EL TIEMPO DE VIAJE EN COCHE - TRATAMIENTOS METRO Y AUTOBÚS

\begin{tabular}{|l|c|c|c|}
\hline & \multicolumn{2}{|c|}{ Proporción de viajeros en coche } \\
\hline & $\leq \mathbf{5 5 \%}$ & $>\mathbf{5 5 \%} \leq \mathbf{7 5 \%}$ & $>\mathbf{7 5 \%}$ \\
\hline $\begin{array}{c}\text { Variación en el tiempo de viaje en coche } \\
\text { (en minutos) }\end{array}$ & 0 & +5 & +10 \\
\hline
\end{tabular}

Si la proporción de viajeros en coche superaba el 55\%, el tiempo de viaje en coche era igual a aquél seleccionado al azar de la distribución de la Tabla 3. Si la proporción estaba entre $55 \%$ y $75 \%$, el tiempo de viaje en coche se incrementaba en cinco minutos, mientras que si superaba el $75 \%$ el tiempo de viaje aumentaba 10 minutos. Consecuentemente, si el porcentaje de viajeros en coche era menor o igual a $55 \%$, los tiempos esperados de viaje en coche eran veinticinco minutos y el coste esperado del viaje igual a 1,5 unidades. En este caso, dado que los sujetos estaban informados que cada cinco minutos de retraso (adelanto) la pérdida (ganancia) monetaria era de 0,5 unidades, el coste del coche era equivalente al coste del metro de 1,0 unidades por un tiempo de viaje fijo de treinta minutos.

En el tratamiento autobús, el componente casual del tiempo real de viaje en coche estaba determinado por una realización aleatoria de la distribución de probabilidad de la Tabla 5 .

TABLA 5

IMPACTO DE FACTORES CASUALES EN EL TIEMPO DE VIAJE EN COCHE TRATAMIENTO AUTOBÚS

\begin{tabular}{|l|c|c|c|c|c|}
\hline & \multicolumn{5}{|c|}{ Tiempo de viaje en coche } \\
\hline $\begin{array}{l}\text { Tiempo de viaje } \\
\text { (en minutos) } \\
\text { Probabilidad }\end{array}$ & 20 & 25 & 30 & 35 & 40 \\
\hline
\end{tabular}

El valor esperado de esta distribución es veintisiete minutos, que era el tiempo esperado de viaje en coche conocido por los sujetos. El impacto de la congestión de tráfico en el tiempo del coche estaba determinado como en el tratamiento metro (Tabla 4). Entonces, el tiempo esperado de viaje en coche era veintisiete minutos si la proporción de viajeros en coche no era mayor que 55\%, treinta y dos minutos si la proporción estaba entre $55 \%$ y $75 \%$, y treinta y siete minutos si la proporción superaba el $75 \%$.

El tiempo de viaje en autobús también se supuso incierto y dependiente de factores casuales y congestión de tráfico. El efecto de los factores causales en el tiempo en autobús era determinado por una realización aleatoria de la distribución de probabilidad de la Tabla 6. 
TABLA 6

IMPACTO DE FACTORES CASUALES EN EL TIEMPO DE VIAJE EN AUTOBÚS - TRATAMIENTO AUTOBÚS

\begin{tabular}{|l|c|c|c|}
\hline & \multicolumn{3}{|c|}{ Tiempo de viaje en autobús } \\
\hline Tiempo de viaje (en minutos) & 30 & 35 & 40 \\
Probabilidad & $70 \%$ & $20 \%$ & $10 \%$ \\
\hline
\end{tabular}

Hemos excluido la posibilidad de que el autobús pudiera llegar con adelanto respecto al tiempo esperado. El valor esperado de la distribución de la Tabla 6 es 32 minutos, que es exactamente el tiempo esperado comunicado a los participantes.

El impacto de la congestión en la ruta en el tiempo de viaje en autobús se supuso menor que en el caso del viaje en coche, por lo cual resulta adecuado cuando el sistema de tráfico incluye carriles o rutas reservadas para el autobús (Tabla 7).

TABLA 7

IMPACTO DE LA CONGESTIÓN DE TRÁFICO EN EL TIEMPO DE VIAJE EN AUTOBÚS - TRATAMIENTO AUTOBÚS

\begin{tabular}{|l|c|c|}
\hline & \multicolumn{2}{|c|}{ Proporción de viajeros en coche } \\
\hline & $\mathbf{5 5 5 \%}$ & $\mathbf{> 5 5 \%}$ \\
\hline Variación en el tiempo de viaje en autobús (en minutos) & 0 & +5 \\
\hline
\end{tabular}

Si la proporción real de viajeros en coche no superaba el 55\%, el tiempo de viaje en autobús era igual al valor determinado por una realización de la distribución de la Tabla 6. Por lo tanto, la congestión de tráfico no tenía ningún impacto en el tiempo de viaje en autobús si la proporción de viajeros en coche era menor al 55\% del total de la población. En este caso, en el tratamiento autobús 1,0 el coste esperado del autobús era igual a una unidad para un tiempo de viaje esperado de treinta y dos minutos, que era equivalente al coste esperado del coche de 1,5 unidades para un tiempo de viaje esperado de veintisiete minutos. Esta equivalencia no se mantenía en el tratamiento autobús 0,8 , en el que el coste fijo del autobús se reducía a 0,8.

Resumiendo, los parámetros que definían el coste real de viaje estaban determinados de la siguiente forma:

a) En el tratamiento metro, el coste esperado de viaje en coche y en metro era equivalente si la proporción de viajeros en coche no superaba el 55\%;

b) En el tratamiento autobús 1,0, los costes esperados de viaje en coche y en autobús eran equivalentes si la proporción de viajeros en coche no superaba el 55\%;

c) En el tratamiento autobús 0,8 , el coste esperado del autobús era $20 \%$ menor que el del coche si la proporción de viajeros en coche no superaba el 55\%. 


\section{Resultados}

Los resultados se discuten en tres secciones que corresponden a los patrones de comportamiento mostrados por los sujetos en las elecciones del medio de transporte.

\section{- La preferencia por el coche}

El coche es generalmente percibido como el medio de transporte que otorga la mayor libertad e independencia. Los costes asociados al uso del coche frecuentemente se subestiman porque no se asocian enteramente al tiempo que se utiliza el vehículo $^{2}$. Estos factores explican la presencia de una propensión generalizada a utilizar el coche y de una resistencia psicológica a reducir su uso (Van Vugt y otros, 1995; Tertoolen y otros, 1998; Hensher, 2001; Bamberg y otros, 2003). Nuestros datos experimentales respaldan esta observación. La Tabla 8 presenta la proporción de elección de coche en todos los tratamientos cada cinco períodos.

TABLA 8

\section{PROPORCIÓN DE ELECCIONES DE COCHE POR TRATAMIENTO} (CADA CINCO PERÍODOS)

\begin{tabular}{|c|c|c|c|}
\hline Período & Metro & Autobús 1,0 & Autobús 0,8 \\
\hline 1 & 0,70 & 0,60 & 0,59 \\
5 & 0,67 & 0,67 & 0,35 \\
10 & 0,60 & 0,47 & 0,35 \\
15 & 0,57 & 0,67 & 0,47 \\
20 & 0,57 & 0,53 & 0,53 \\
25 & 0,77 & 0,53 & 0,41 \\
30 & 0,67 & 0,73 & 0,71 \\
35 & 0,70 & 0,60 & 0,71 \\
40 & 0,60 & 0,53 & 0,53 \\
45 & 0,67 & 0,60 & 0,53 \\
50 & 0,73 & 0,53 & 0,53 \\
\hline Total & $\mathbf{0 , 6 8}$ & $\mathbf{0 , 5 8}$ & $\mathbf{0 , 5 0}$ \\
\hline
\end{tabular}

En el tratamiento metro, el porcentaje de sujetos que eligen el coche está raramente por debajo del 55\%. Aún más significativo es que este porcentaje es casi el mismo en el primer período (70\%) que en el último (73\%), lo que respalda la hipótesis de que las elecciones dependen más de una propensión inicial a utilizar el coche que del aprendizaje durante el experimento. La Figura 1 muestra la proporción de viajeros en coche en las dos sesiones de metro.

\footnotetext{
${ }^{2}$ Los costes del coche están dados por los costes directos diarios tales como combustible, costes de aparcamiento y por los costes indirectos por desgaste de cubiertas, mantenimiento, etc. Además, las externalidades tales como la polución o los costes sociales debidos a accidentes de tráfico no son fácilmente calculables y usualmente no son tenidos en cuenta.
} 
FIGURA 1

PROPORCIÓN DE VIAJEROS EN COCHE

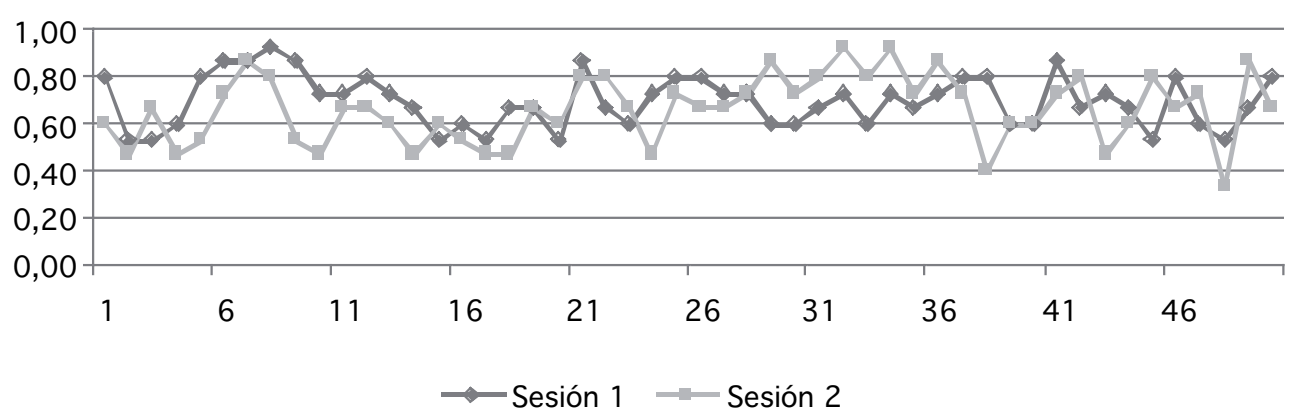

Vale la pena destacar que la proporción de viajeros en coche alcanza su máximo alrededor del $7 .^{\circ}-8 .^{\circ}$ período, luego se reduce en diez períodos y vuelve a aumentar más adelante. Dicho patrón de comportamiento parece depender más de inclinaciones del comportamiento adquiridas fuera del laboratorio que del contexto específico de aprendizaje. Esta interpretación se confirma observando los costes esperados y reales de viaje. Como se señaló anteriormente, las distribuciones de probabilidad que determinan los efectos casuales y de la congestión de tráfico son tales que el coste del coche es mayor que el coste del metro si la proporción de viajeros en coche es mayor que el $55 \%$. El porcentaje de usuarios de coche es menor que el límite de congestión solamente en 10 de los 100 períodos. Consecuentemente, los costes esperados del metro son menores que los esperados del coche en los 90 períodos restantes. En lo que concierne a los costes reales determinados por las elecciones de los sujetos y las realizaciones aleatorias, el coste promedio del coche para todos los períodos es igual a 1,46 , que es mayor que el coste fijo del metro $(1,00)$. A pesar de esta ventaja comparativa para el metro, la razón entre el usuario de coche y metro es aproximadamente dos a uno.

En el tratamiento autobús $(1,0)$, en promedio, más de la mitad de los sujetos elige el coche (Figura 2). 
FIGURA 2

PROPORCIÓN DE VIAJEROS EN COCHE - AUTOBÚS VS. COCHE

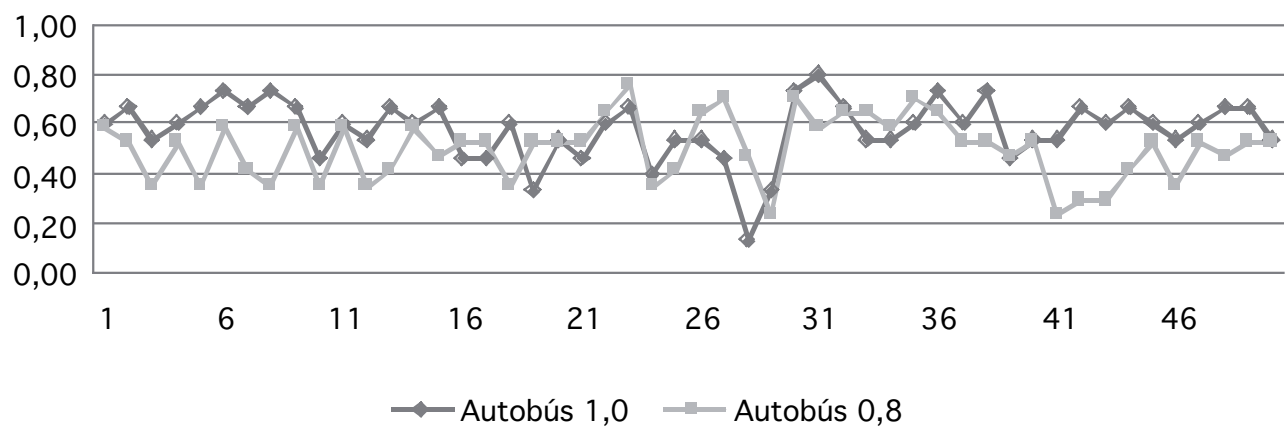

En este caso, la equivalencia entre los costes esperados de las dos alternativas de transporte disponibles se mantiene por debajo del límite de 55\% de usuarios de coche, pero esta proporción fue superada en 40 de los 50 períodos. La preferencia por el coche se confirma también cuando se consideran los costes reales. En promedio, el coste del coche $(1,63)$ es mayor que el del autobús $(1,21)$. La Figura 3 muestra los costes reales del autobús y del coche en el tratamiento autobús 1,0.

\section{FIGURA 3}

\section{COSTES REALES DE VIAJE. TRATAMIENTO AUTOBÚS 1,0}

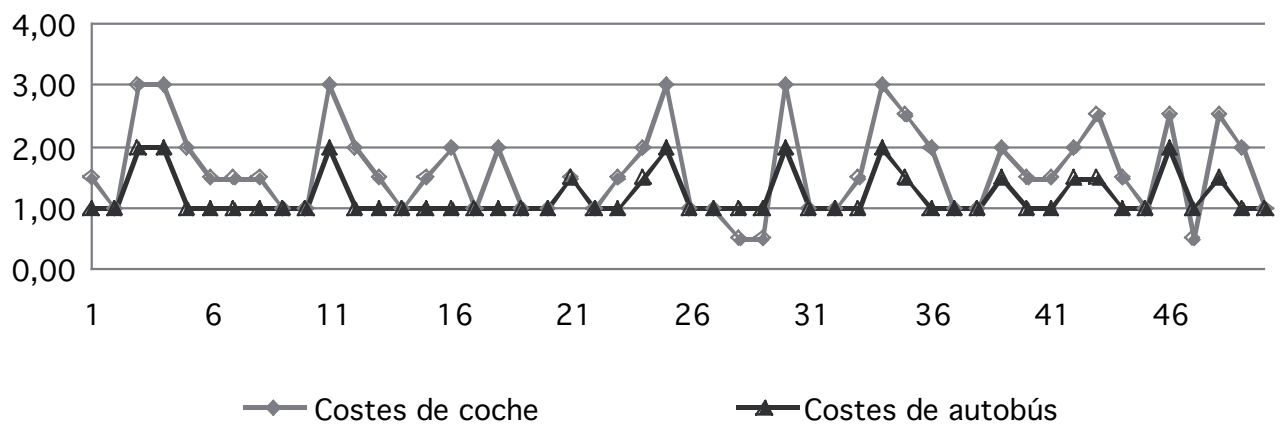


En el tratamiento autobús 0,8 , la reducción del coste fijo del autobús aumenta significativamente el porcentaje de viajeros en autobús, pero el porcentaje de viajeros en coche es consistentemente menor que la mitad de la población solamente en los primeros veinte períodos y durante la mitad de la sesión (Figura 2). A lo largo de todos los períodos, la proporción de usuarios de coche es exactamente $50 \%$. Además, en este tratamiento, los costes reales validan la robustez de la preferencia por el coche, dado que los costes reales del autobús son $19 \%$ menores que los del coche $(0,96$ vs. 1,48). La Figura 4 muestra los costes reales del autobús y del coche en el tratamiento autobús 0,8 .

\section{FIGURA 4}

\section{COSTES REALES DE VIAJE. TRATAMIENTO AUTOBÚS 0,8}

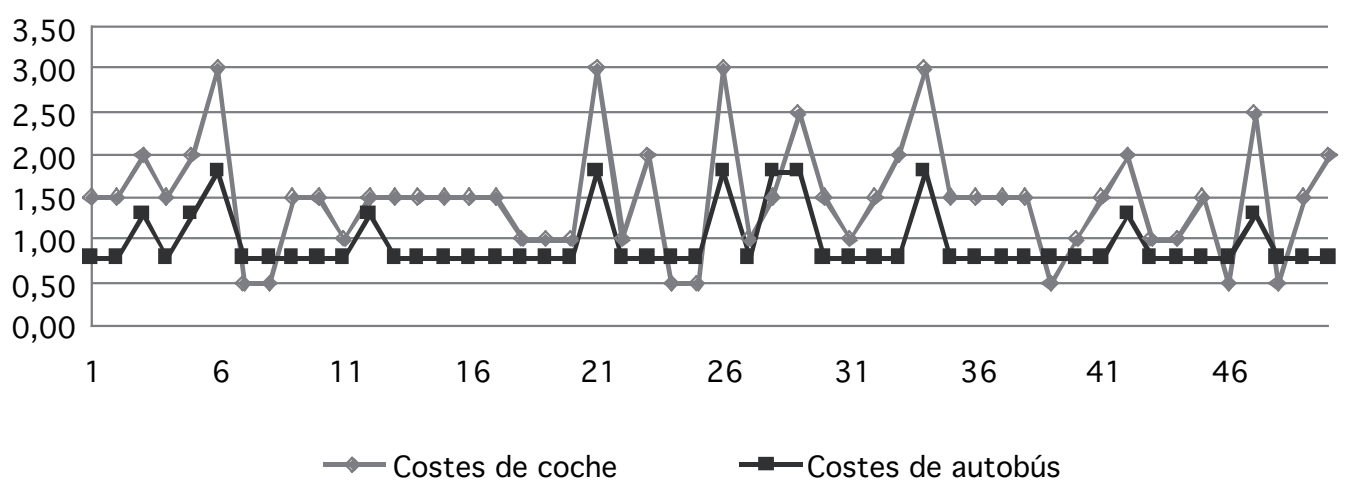

La dinámica de las elecciones confirma la existencia de una inclinación positiva hacia el uso del coche. Después de ciertos períodos en los que el coste del coche es muy elevado los sujetos reaccionan rápidamente, pero los efectos disuasorios no perduran por mucho tiempo. La Tabla 9 muestra la proporción de usuarios de coches en períodos posteriores a un valor inusualmente alto de coste del coche. 
TABLA 9

PROPORCIÓN DE USUARIOS DE COCHE CON POSTERIORIDAD A ELEVADOS COSTES DEL COCHE

\begin{tabular}{|c|c|c|c|}
\hline Tratamiento / Sesión & Período & $\begin{array}{l}\text { Coste del } \\
\text { coche }\end{array}$ & $\begin{array}{c}\text { Proporción de } \\
\text { usuarios de coche }\end{array}$ \\
\hline Metro vs. Coche - Sesión 1 & $\begin{array}{l}34 \\
35 \\
36 \\
37 \\
38 \\
39 \\
40 \\
41\end{array}$ & $\begin{array}{l}3,00 \\
1,50 \\
1,00 \\
1,00 \\
3,00 \\
2,50 \\
1,00 \\
1,00\end{array}$ & $\begin{array}{l}0,73 \\
0,67 \\
0,73 \\
0,80 \\
0,80 \\
0,60 \\
0,60 \\
0,87\end{array}$ \\
\hline Metro vs. Coche - Sesión 2 & $\begin{array}{l}46 \\
47 \\
48 \\
49\end{array}$ & $\begin{array}{l}2,50 \\
2,50 \\
1,00 \\
1,50\end{array}$ & $\begin{array}{l}0,67 \\
0,73 \\
0,33 \\
0,87\end{array}$ \\
\hline Autobús 1,0 vs. Coche - Sesión 3 & $\begin{array}{l}25 \\
26 \\
27 \\
28 \\
29 \\
30\end{array}$ & $\begin{array}{l}3,00 \\
1,00 \\
1,00 \\
0,50 \\
0,50 \\
3,00\end{array}$ & $\begin{array}{l}0,53 \\
0,53 \\
0,47 \\
0,13 \\
0,33 \\
0,73\end{array}$ \\
\hline Autobús 0,8 vs. Coche - Sesión 4 & $\begin{array}{l}6 \\
7 \\
8 \\
9\end{array}$ & $\begin{array}{l}3,00 \\
0,50 \\
0,50 \\
1,50\end{array}$ & $\begin{array}{l}0,59 \\
0,41 \\
0,35 \\
0,59\end{array}$ \\
\hline
\end{tabular}

La proporción retorna al valor inicial en no más de dos o tres períodos. Esta reacción es particularmente evidente en la secuencia 34-41 de la Sesión 1. Después de dos picos de tres unidades en los costes, la proporción de usuarios de coche cae inmediatamente, pero vuelve a niveles altos en después de sólo tres períodos. En la Tabla 9 se muestran grupos de períodos de las diferentes fases de todas las sesiones donde esto ocurre.

Finalmente, se destaca que el coche es más comúnmente elegido cuando el medio de transporte alternativo es el metro que cuando es el autobús $(t=4,708, p<0,05$ en el Tratamiento Autobús 1,0; $\mathrm{t}=7,86, \mathrm{p}<0,05$ en el Tratamiento Autobús 0,8$)$. La Tabla 10 confirma este resultado mostrando la proporción de elecciones de coche en todos los períodos. 
TABLA 10

FRECUENCIAS DE PERÍODOS POR PORCENTAJES Y TRATAMIENTOS (TODOS LOS PERÍODOS)

\begin{tabular}{|c|c|c|c|}
\hline $\begin{array}{c}\text { Proporción de } \\
\text { usuarios de coche }\end{array}$ & Coche vs. Metro & Coche vs. Autobús 1,0 & Coche vs. Autobús 0,8 \\
\hline$<0,50$ & 10 & 10 & 20 \\
$\geq 0,50<0,60$ & 10 & 11 & 20 \\
$\geq 0,60<0,70$ & 34 & 23 & 5 \\
$\geq 0,70<0,80$ & 18 & 5 & - \\
$\geq 0,80<0,90$ & 25 & 1 & - \\
$\geq 0,90$ & 3 & - & $\mathbf{5 0}$ \\
\hline Total de períodos & $\mathbf{1 0 0}$ & $\mathbf{5 0}$ & - \\
\hline
\end{tabular}

En el tratamiento metro, la proporción de usuarios de coche es menor que 0,50 solamente en $10 \%$ de los períodos, mientras que este porcentaje aumenta a $20 \%$ en el tratamiento autobús 1,0 y a $40 \%$ en el tratamiento autobús 0,8 .

Una explicación plausible de este resultado viene dada por la teoría prospectiva. Según el modelo de comportamiento de Kahneman y Tversky (1973), en un contexto de decisión en el cual solamente son posibles las pérdidas, la gente exhibe una actitud de búsqueda de riesgo. Como se discutió anteriormente, la investigación de laboratorio respalda este modelo de elección de ruta (Katsikopoulos y otros, 2000). En nuestro experimento, el coche sería preferido al metro por ser un medio de transporte más arriesgado. Esta propensión decrecería en los tratamientos autobús debido a que ambas alternativas de viaje son inciertas.

\section{- El efecto de la primera elección}

Una inspección visual de las Figuras 1 y 2 permite apreciar que la proporción de usuarios de coche aumenta en la segunda mitad de cada tratamiento y se hace menos errática en todos los tratamientos. El análisis estadístico valida esta idea. La proporción media de usuarios de coche a lo largo de todos los períodos y tratamientos es aproximadamente la misma: aumenta de 0,57 en los primeros 25 períodos a 0,59 en los últimos 25 períodos, mientras que la desviación típica se reduce de 0,180 a 0,145 . Además, el número de sujetos que cambian de medio de transporte en los últimos 15 períodos es $1 / 3$ menor que durante los primeros 15 períodos.

Estos datos podrían interpretarse como evidencia de aprendizaje: los sujetos irían descubriendo gradualmente las distribuciones de probabilidad que determinan los tiempos de viaje y este proceso reduciría la variabilidad de las elecciones. Sin embargo, el comportamiento de los sujetos exhibe una dependencia significativa de la primera elección individual. La Figura 5 muestra el porcentaje de sujetos por elecciones de metro, divididos entre los que eligen el coche y los que eligen el metro en el primer período. 


\section{FIGURA 5}

EFECTO DE LA PRIMERA ELECCIÓN - TRATAMIENTO METRO

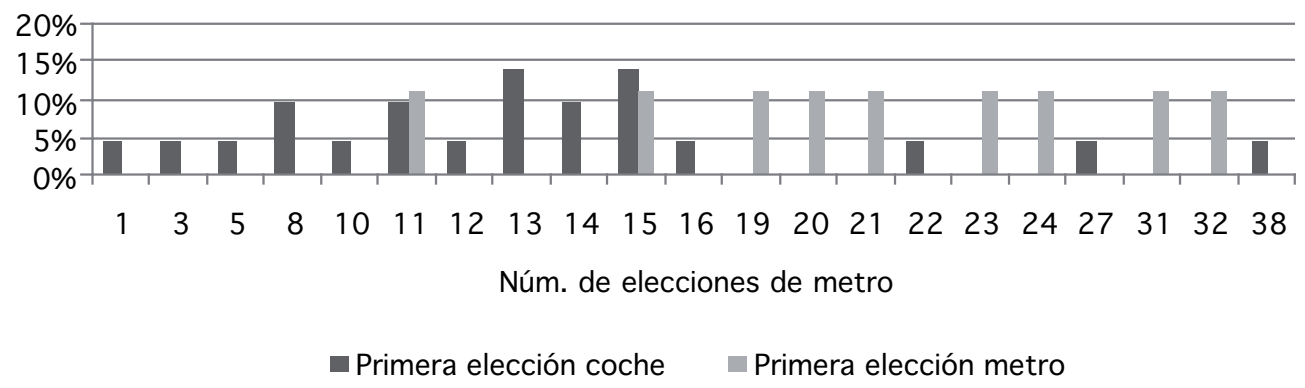

Los sujetos que eligen el coche en el primer período están concentrados en la parte inferior de la distribución: $86 \%$ de los viajeros que comienzan con el coche eligen el metro menos de 16 veces, mientras que solamente $22 \%$ de quienes comienzan con el metro prefieren el metro menos de 16 veces. En todos los períodos, los sujetos que comienzan con el coche prefieren el metro con una probabilidad del $27 \%$ y eligen el metro, en promedio, 13,8 veces, mientras que quienes eligen inicialmente el metro confirman esta preferencia con una probabilidad de $43 \%$ y eligen el metro, en promedio, 21,7 veces. La Figura 6 presenta información sobre el efecto de la primera elección en el tratamiento autobús.

La probabilidad de que quienes comienzan con el autobús prefieran el autobús en todos los períodos es $27 \%$, mientras que la misma probabilidad para aquéllos que comienzan con el coche es $19 \%$. El 80\% de quienes comienzan con el coche eligen el autobús menos de 28 veces versus $60 \%$ de aquéllos que exhiben una preferencia por el autobús al comienzo del experimento.

Esta observación respalda la hipótesis de que la primera elección de los sujetos es una señal confiable de su propensión a preferir un cierto medio de transporte. Es como si los sujetos llevaran al laboratorio las preferencias aplicadas a elecciones reales y se adhirieran a ellas con una probabilidad elevada. Este resultado contrasta con un enfoque correcto de la experimentación en economía. Uno de los principios básicos de la metodología de laboratorio es que el uso de sujetos no profesionales e incentivos monetarios permite hacer las características innatas de los sujetos en gran parte irrelevantes. En nuestro diseño experimental, este objetivo parece cumplirse sólo parcialmente. La referencia explícita a los medios de transporte revela no solamente que los sujetos exhiben una evidente preferencia por el coche, sino además que sus preferencias están inicialmente sesgadas hacia una de las alternativas y que 


\section{FIGURA 6}

EFECTO DE LA PRIMERA ELECCIÓN - TRATAMIENTO AUTOBÚS

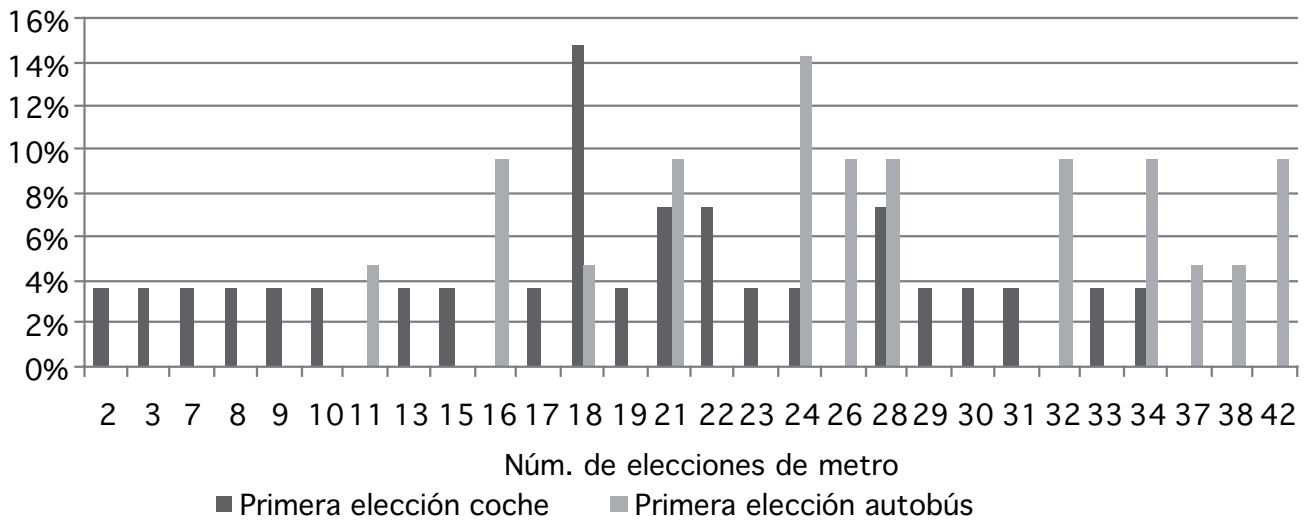

esta tendencia tiende a anular el efecto de los incentivos. El comportamiento de los sujetos depende más del aprendizaje previo fuera del laboratorio que de las ganancias esperadas en el laboratorio.

Este fenómeno puede ser explicado teniendo en cuenta la clase de decisión que los sujetos toman. Las elecciones del medio de transporte son decisiones rutinarias, tomadas una y otra vez. Progresivamente se vuelven elecciones habituales, y dejan de ser consecuencia de un proceso deliberado de ponderación de pros y contras (Aarts y otros, 1997). En el laboratorio, esta actitud se desencadena automáticamente por las señales dadas a los sujetos. Una vez que el acto de elegir un medio de transporte se produce, el razonamiento enfocado a una meta directa juega un papel limitado en la determinación de las decisiones.

Esta interpretación, que enfatiza las limitaciones en la capacidad de procesar exhaustivamente la información disponible, se ve corroborada observando la relación entre las elecciones de los sujetos y los tiempos de viaje experimentados. La Tabla 11 presenta los coeficientes de correlación entre el número de elecciones de coche y el coste medio del coche, en todos los períodos previos, en los diez períodos previos y en los cinco períodos previos, respectivamente. 
TABLA 11

COEFICIENTES DE CORRELACIÓN ENTRE ELECCIONES Y COSTES DEL COCHE

\begin{tabular}{|l|c|c|c|}
\hline \multicolumn{1}{|c|}{ Tratamiento } & $\begin{array}{c}\text { N. de elecciones } \\
\text { de coche en el } \\
\text { tiempo } / \text { Costes } \\
\text { medios del coche } \\
\text { en todos los } \\
\text { períodos previos }\end{array}$ & $\begin{array}{c}\text { N. de elecciones } \\
\text { de coche en el } \\
\text { tiempo t / Costes } \\
\text { medios del coche } \\
\text { en todos los } \\
\text { períodos previos }\end{array}$ & $\begin{array}{c}\text { N. de elecciones } \\
\text { de coche en el } \\
\text { tiempo t / Costes } \\
\text { medios del coche } \\
\text { en todos los } \\
\text { períodos previos }\end{array}$ \\
\hline $\begin{array}{l}\text { Metro vs. Coche } \\
\text { Autobús } 1,0 \text { vs. Coche } \\
\text { Autobús } 0,8 \text { vs. Coche }\end{array}$ & $-0,049$ & $-0,207$ & $-0,200$ \\
$-0,195$ & $-0,391$ & $-0,251$ \\
\hline
\end{tabular}

La correlación entre los usuarios de coche y los costes del coche durante los cinco y diez períodos anteriores es mayor que la correlación con los costes del coche en todos los períodos previos. En consecuencia, las expectativas de los viajeros con respecto a los tiempos de viaje futuros se actualizan imperfectamente. Los viajeros exhiben una memoria limitada, tal como lo postula la investigación psicológica sobre la capacidad de la memoria de trabajo (Kareev y otros, 1997). Incluso si los viajeros se adhieren al principio de la maximización de los pagos, su incapacidad para considerar en el análisis todos los costes de viaje previos les hace computar incorrectamente su mejor estrategia racional.

\section{- Rigidez del medio de transporte}

La existencia de un efecto de la primera elección debería implicar que los sujetos exhiben una aversión a cambiar el medio de transporte. Solamente $28,6 \%$ de los sujetos en el tratamiento metro y $39 \%$ en los tratamientos autobús cambian más de 20 veces en 50 períodos. En promedio, los sujetos cambian de medio 17,7 veces en el tratamiento metro y 18,0 veces en los tratamientos autobús. Las Figuras 7 y 8 presentan la distribución de los sujetos según el número de veces que cambian de medio de transporte en los tratamientos metro y autobús. 
FIGURA 7

NÚM. DE CAMBIOS EN EL MEDIO DE TRANSPORTE TRATAMIENTO METRO

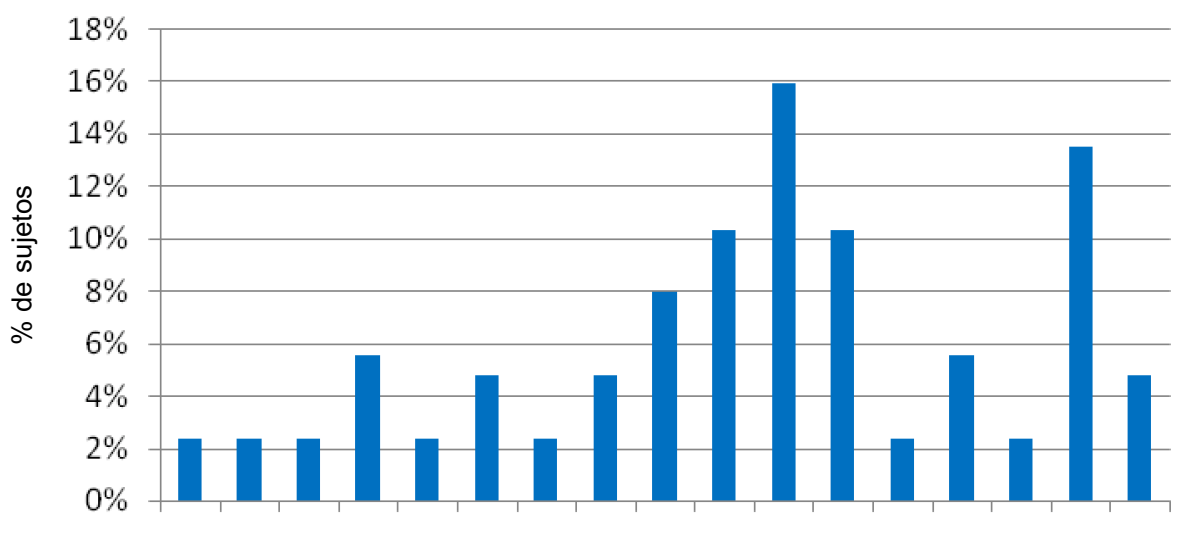

$\begin{array}{lllllllllllllllll}2 & 4 & 6 & 7 & 8 & 9 & 12 & 14 & 16 & 18 & 19 & 20 & 22 & 23 & 24 & 25 & 32\end{array}$

FIGURA 8

NÚM. DE CAMBIOS EN EL MODO DE VIAJE TRATAMIENTO AUTOBÚS

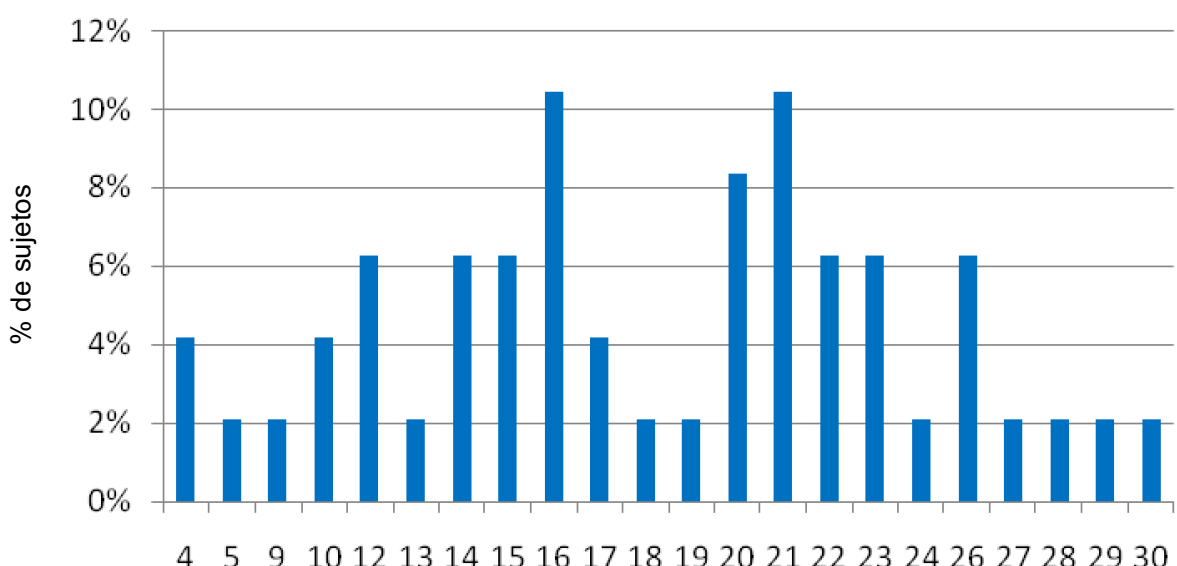

El análisis de los datos confirma que los cambios están negativamente correlacionados con el tiempo de viaje promedio en ambos tratamientos, aunque la relación no es estadísticamente significativa. Sin embargo, en Selten y otros (2007) se presenta evidencia de una relación positiva entre la rigidez en la ruta y los pagos totales en el caso de la elección de ruta.

Selten y otros (2007) analizan además cómo los viajeros tienen en cuenta las elecciones de otros viajeros. Estos autores encuentran dos tipos diferentes de respuesta. En el primer tipo, llamado respuesta directa, un viajero cambia de medio en el período siguiente si los tiempos de viaje en el período previo son relativamente 
altos. En el segundo tipo, llamado respuesta contraria, los sujetos cambian de medio si los tiempos de viaje en el período previo son relativamente bajos, sobre la base de la expectativa de que este medio atraerá más viajeros en el período siguiente y, consecuentemente, producirá más congestión de tráfico.

Para investigar la ocurrencia de estos dos tipos de respuesta en nuestro experimento, definimos un coste de medio de transporte como relativamente alto si es mayor que el coste de viaje promedio en los diez períodos anteriores y como relativamente bajo si es menor ${ }^{3}$. En nuestro diseño, la congestión de tráfico depende solamente de la proporción de usuarios de coche y los sujetos observan los costes del tiempo de viaje en todos los medios. En consecuencia, adoptamos las definiciones de respuesta directa o contraria tal como se establece en la Tabla 12.

TABLA 12

\section{DEFINICIONES DE MODO DE RESPUESTA DIRECTA} Y CONTRARIA

\begin{tabular}{|l|c|c|}
\hline \multirow{2}{*}{ Elecciones } & \multicolumn{2}{|c|}{ Costes de viaje } \\
\cline { 2 - 3 } & Alto & Bajo \\
\hline Cambia de coche a metro/autobús & Directa (d1) & Contraria (c1) \\
Cambia de metro/autobús a coche & Contraria (c1) & Directa (d1) \\
Continúa en coche & Contraria (c2) & Directa (d2) \\
Continúa en metro/autobús & Directa (d2) & Contraria (c2) \\
\hline
\end{tabular}

Por ejemplo, en el tratamiento metro, un cambio en un medio de transporte es una respuesta directa si el sujeto cambia de coche a metro después de observar un coste del coche relativamente alto o cambia de metro a coche después de observar un coste del coche relativamente bajo, mientras que es una respuesta contraria si cambia de coche a metro después de observar un coste de viajar en coche relativamente bajo o cambia de metro a coche después de observar un coste del coche relativamente alto. Confirmar el metro como medio de transporte durante dos períodos consecutivos es una respuesta directa si los costes del coche son relativamente altos y una respuesta contraria si los costes del coche son relativamente bajos.

Entonces, para cada sujeto $i$ calculamos el coeficiente de Yule-Hamman $\mathrm{H}_{\mathrm{i}}$, que en nuestro experimento se obtiene de la siguiente manera:

$$
H_{i}=\frac{\sum_{j=1}^{n} \text { Directa }_{i j}-\sum_{j=1}^{n} \text { Contraria }_{i j}}{\sum_{j=1}^{n}\left(\text { Directa }_{j}+\text { Contraria }_{j}\right)}
$$

3 Suponemos que los sujetos toman sus decisiones sobre la base de los diez períodos anteriores, tal como se señala en la Tabla 10, en la cual la correlación entre el número de usuarios de coche y el coste medio del coche durante los diez períodos previos es mayor que la correlación con los costes medios del coche durante todos los períodos. 
El coeficiente $H_{\mathrm{i}}$ varía entre $-1 \mathrm{y}+1$, de forma que los valores positivos y altos indican el predominio del modo de respuesta directa, mientras que valores negativos y altos (en valor absoluto) indican el predominio del modo de respuesta contraria. Si los valores están concentrados alrededor del cero ningún modo predomina sobre el otro. Las Figuras 9 y 10 muestran el coeficiente $\mathrm{H}$ para todos los sujetos en los tratamientos metro y autobús, respectivamente.

FIGURA 9

COEFICIENTE DE YULE-HAMMAN - TRATAMIENTO METRO

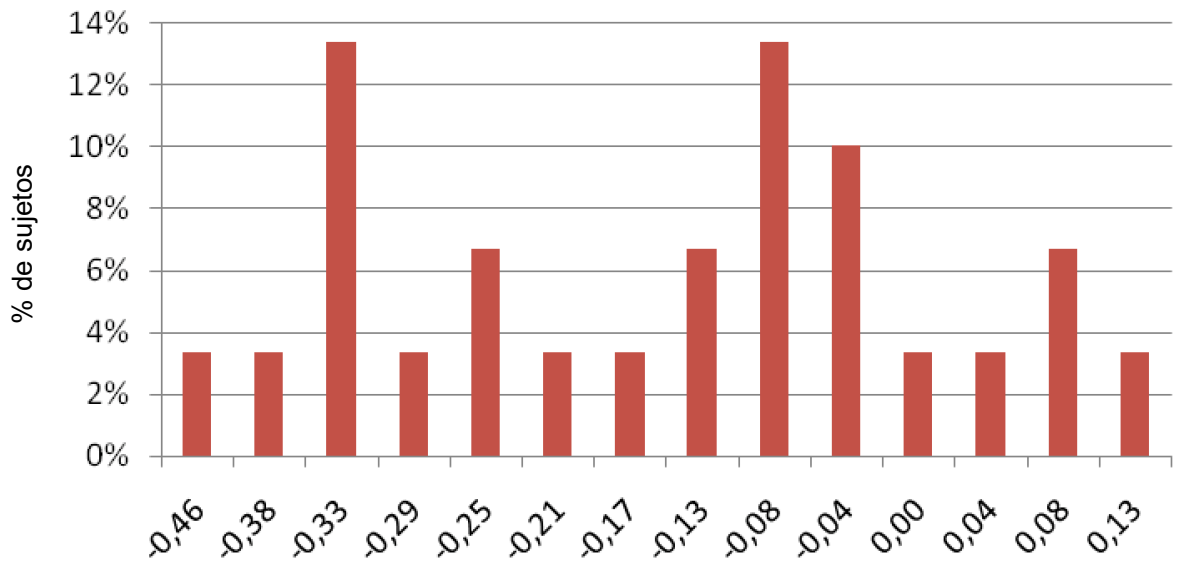

FIGURA 10

COEFICIENTE DE YULE-HAMMAN - TRATAMIENTO AUTOBÚS

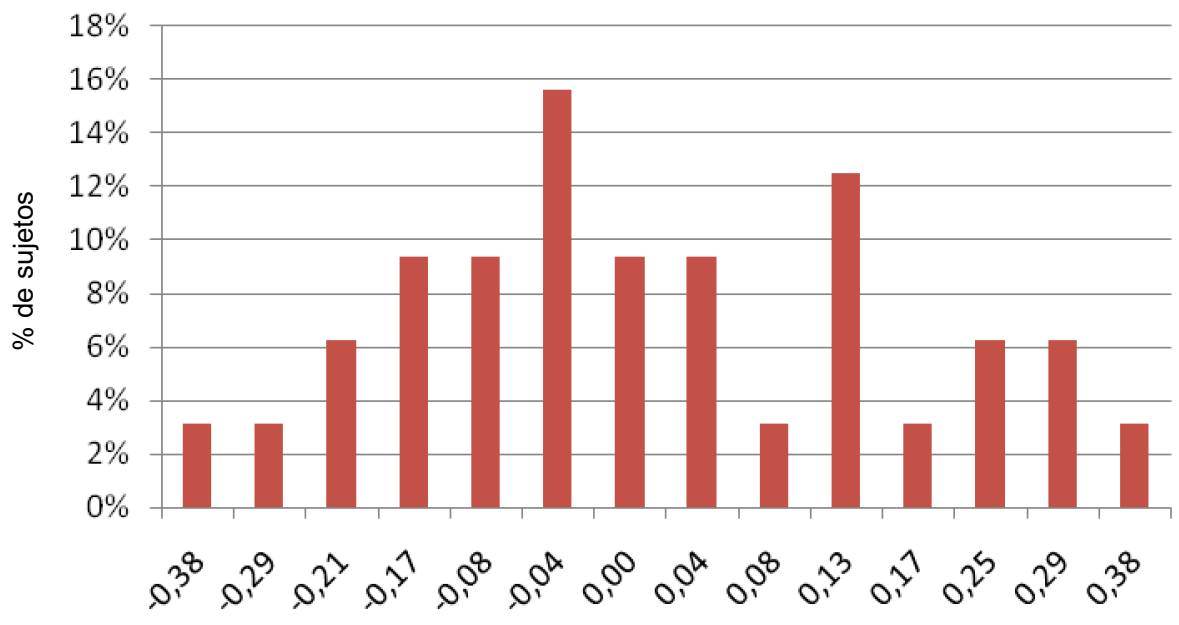


En el tratamiento metro, el coeficiente de Yule-Hamman muestra una suave tendencia hacia el modo de respuesta contraria. Sin embargo, el coeficiente está concentrado en su mayor parte alrededor de cero en ambas distribuciones: varía entre $-0,46$ y $+0,13$ en el tratamiento metro y entre $\pm 0,38$ en los tratamientos autobús. De acuerdo a la clasificación propuesta por Selten y otros (2007), en nuestro experimento ningún sujeto puede ser clasificado como respondente directo o contrario, que sería el caso si el coeficiente de Yule-Hamman fuera menor que $-0,5$ o mayor que $+0,5$, respectivamente.

Una interpretación posible de este resultado es que el efecto de la primera elección reduce la tendencia a cambiar el medio de transporte. La propensión a la rigidez en la elección del medio de transporte se corrobora por el hecho de que los sujetos en el rango medio de las distribuciones de las Figuras 9 y 10 cambian de medio de transporte, en promedio, menos que aquéllos situados en los cuartiles extremos. Esta evidencia confirma que la conducta de los sujetos está más estrictamente relacionada con aprendizaje previo fuera del laboratorio que con aprendizaje durante el experimento.

\section{Conclusiones}

Nuestro estudio presenta evidencia experimental de que los viajeros determinan las elecciones de medio de transporte mediante el uso de procedimientos heurísticos que pueden dar lugar a desviaciones robustas de la minimización de los tiempos de viaje. Encontramos que los sujetos exhiben una marcada preferencia por el coche, son propensos a mantener invariante el medio de transporte y, más específicamente, confirman su primera elección y actualizan sus expectativas sobre los tiempos de viaje imperfectamente. La importancia de estos resultados se amplifica por el hecho de que en nuestro experimento los viajeros contaban no solamente con información adquirida a través de la experiencia personal sino también con los tiempos de viaje reales en los medios alternativos.

Estos resultados tienen implicaciones relevantes para el diseño de políticas de transporte. Primero, se refuta la creencia de que la provisión de información más precisa y exhaustiva a los viajeros mejora necesariamente la racionalidad de sus elecciones. Las elecciones de medio de transporte están determinadas por una variedad de factores psicológicos tales como el hábito, memoria imperfecta, preferencia por un determinado medio, que deben ser analizados y tenidos en cuenta para mejorar la eficiencia de las redes de transporte. En particular, el uso habitual del coche parece ser resistente al efecto de los incentivos económicos. Consecuentemente, es escaso el progreso que se puede esperar de solicitar a los viajeros individuales que reduzcan voluntariamente el uso del coche. Debido a las dificultades dominantes para cambiar la percepción cognitiva individual de las elecciones de medio de transporte, las políticas tienen que elaborar diferentes estrategias para ser efectivas. Una variedad de señales de poca intensidad (tales como incrementos progresivos en los costes del combustible, impuestos diferenciados, derechos de aparcamiento) no se espera que 
sean efectivas, al tiempo que se necesita una acción masiva para que sea correctamente percibida por los individuos. Además, la propensión al riesgo surgida como un aspecto predominante en un juego de pérdida y el respeto a los cronogramas de tiempo resultaron ser menos importantes que la posibilidad aleatoria de ahorrar tiempo utilizando el coche. Nuestros resultados experimentales además son útiles para señalar diferentes motivaciones que usualmente determinan las elecciones de viaje: las conveniencias (monetarias y de tiempo), la propensión (actitud individual y expectativas) y las funciones (motivaciones para viajar). Éstas revelan que los efectos de los incentivos económicos pueden quedar anulados por factores casuales y actitudes subjetivas. Esta observación puede además llevarnos a la notable conclusión de apoyar acciones definitivas tales como prohibiciones, en ausencia de medidas efectivas de incentivos para lograr los comportamientos esperados.

\section{Referencias}

[1] AARTS, H., VERPLANKEN, B., y van KNIPPENBERG, A. (1997). Habit and information use in travel mode choices, Acta Psychologica, 96, pp. 1-14.

[2] ABDEL-ATY, M., y ABDALLAH, M.H. (2004). Modeling drivers' diversion from normal routes under ATIS using generalized estimating equations and binomial probit link function, Transportation, 31, pp. 327-348.

[3] AVINERI, E., y PRASHKER, J. N. (2003). Sensitivity to Uncertainty: the need for a paradigm shift, Transportation Research Record, 1854, pp. 90-98.

[4] AVINERI, E., y PRASHKER, J. N. (2006). The impact of travel time information on travelers' learning under uncertainty, Transportation, 33, pp. 393-408.

[5] BAMBERG, S., RÖLLE, D., y WEBER, C. (2003). Does habitual car use not lead to more resistance to change of travel mode? Transportation, 30, pp. 97-108.

[6] BEN-ELIA, E., EREV, I., y SHIFTAN, Y. (2008). The combined effect of information and experience on drivers' route-choice behavior, Transportation, 35, pp. 165-177.

[7] CASCETTA, E. (1989). A Stochastic Process Approach to the Analysis of Temporal Dynamics in Transportation Networks, Transportation Research-B, 23, pp. 1-17.

[8] FISCHBACHER, U. (2007). «z-Tree: Zurich toolbox for readymade economic experiments. Experimenter's manual», Experimental Economics 10, 2, pp. 171-178.

[9] FRIESZ, T. L., BERNSTEIN, D., MEHTA, N. J., TOBIN, R. L., y GANJALIZADEH, S. (1994). Day-to-day dynamic network disequilibria and idealized traveler information systems, Operations Research, 42, pp. 1120-1136.

[10] GÄRLING, T. (1998). Behavioural assumptions overlooked in travel-choice modeling. En: J. Ortuzar, S. Jara-Diaz, D. Hensher (eds.), Travel Behaviour Research: Updating the State of Play, Pergamon, Oxford, UK: Elsevier, pp. 1-18.

[11] HENSHER, D. A. (2001). The sensitivity of the valuation of travel time saving to the specification of unobserved effects, Transportation Research-E, 37, pp. 129-142

[12] KAHNEMAN, D., y TVERSKY, A. (1973). On the psychology of prediction. Psychological Review, 80, pp. 237-251.

[13] KAREEV, Y, ARNON, S., y HOROWITZ-ZELIGER, R. (2002). On the misperception of variability. Journal of Experimental Psychology: General, 131, pp. 287-297. 
[14] KAREEV, Y., LIEBERMAN, I., y LEV, M. (1997). Through a narrow window: Sample size and the perception of correlation. Journal of Experimental Psychology: General, 126, pp. 278-287.

[15] KATSIKOPOULOS, K. V., DUSE-ANTHONY, Y., FISHER, D. L., y DUFFY, S. A. (2000). The framing of drivers' route choices when travel time information is provided under varying degrees of cognitive load. Human Factors, 42, pp. 470-481.

[16] MAHMASSANI, H. S. (1996). Dynamics of commuter behaviour: Recent research and continuing challenges, en M. Lee Gosselin, P. Stopher (eds.), Understanding Travel Behaviour in an Era of Change, Pergamon, Oxford, pp. 279-313.

[17] MAHMASSANI, H. S., y CHANG, G. L. (1985). Dynamic aspects of departure time choice behavior in a commuting system: theoretical framework and experimental analysis. Transportation Research Record, 1037, pp. 88-101.

[18] MAHMASSANI, H., y LIU, Y. (1998). Dynamics of commuting decision behavior under advanced traveler information systems, Transportation Research C, 7, pp. 91-107.

[19] MIRCHANDANI, P., y SOROUSH, H. (1987). Generalized traffic equilibrium with probabilistic travel times and perceptions. Transportation Science, 3, pp. 133-151.

[20] SCHEINER, J., y HOLZ-RAU, C. (2007). Travel mode choice: Affected by objective or subjective determinants?, Transportation Research Record, 34, pp. 487-512.

[21] SELTEN, R., CHMURA, T., PITZ, T., KUBE, S., y SCHRECKENBERG, M. (2007) Commuters route choice behaviour, Games and Economic Behavior, 58, pp. 394-406.

[22] SRINIVASAN, K.K., y MAHMASSANI, H.S. (1999). Role of congestion and information in tripmakers' dynamic decision processes: an experimental investigation, Transportation Research Record, 1676, pp. 43-52.

[23] STARMER, C. 2000 Developments in non-expected utility theory: The hunt for a descriptive theory of choice under risk, Journal of Economic Literature, 38, pp. 332-382.

[24] TERTOOLEN, G., VAN KREVELED, D., y VERSTRATEN, B. (1998). Psychological resistance against attempts to reduce private car use, Transportation Research-A, 32, pp. 171-181.

[25] VAN VUGT, M., MEERTENS, R. M., y VAN LANGE, P. A. M. (1995). Car versus public transportation? The role of social value orientations in a real-life social dilemma. Journal of Applied Social Psychology, 25, pp. 258-278.

[26] VERPLANKEN, B., y AARTS, H. (1999). Habit, attitude, and planned behaviour: Is habit an empty construct or an interesting case of goal-directed automaticity? En W. Stroebe y M. Hewstone (eds.), European Review of Social Psychology, Chichester, England, Wiley, pp. 101-134.

[27] VERPLANKEN, B., AARTS, H., VAN KNIPPENBERG, A., y VAN KNIPPENBERG, C. (1994). Attitude versus general habit: antecedents of travel mode choice, Journal of Applied Social Psychology, 24, pp. 285-300. 


\section{APÉNDICE \\ INSTRUCCIONES (TRADUCIDAS DEL ITALIANO)}

\section{Tratamiento Metro vs. Coche}

\section{BIENVENIDOS AL LABORATORIO}

Este es un experimento sobre toma de decisiones en economía. El IRPET (Instituto Regional para el Planeamiento Económico de la Toscana) y la Universidad de Siena han proporcionado los fondos para realizar esta investigación.

Cada uno de ustedes recibirá un premio de 5 euros por participar. Dependiendo de sus decisiones de inversión pueden obtener ganancias adicionales. Deberán seguir un conjunto de instrucciones para tomar sus decisiones. El experimento es individual: tanto sus elecciones como sus ganancias serán confidenciales para todos excepto para ustedes mismos. El pago final se calcula automáticamente al final del experimento y se les pagará en efectivo al final de la sesión. Pueden comprobar sus resultados al final del experimento.

Para garantizar los mejores resultados del experimento es necesario que respondan de forma individual, sin consultar a otros participantes.

Está permitido hacer preguntas después de que terminemos de repasar esta documentación con ustedes y/o justo antes de comenzar con el experimento.

Habrá sesiones de prueba para mejorar su habilidad para tomar las decisiones antes de comenzar con el experimento real. Si están listos, sigan las instrucciones del experimento que aparecen más abajo.

\section{INSTRUCCIONES}

La decisión que cada uno de ustedes deberá tomar es elegir un medio de transporte. Están disponibles dos medios: metro y coche. El tiempo de viaje en metro está fijo e igual a 30 minutos, mientras que el tiempo esperado de viaje en coche es incierto y, en promedio, es igual a 25 minutos. Después de tomar una decisión, se les informará del tiempo y de los costes reales de su viaje. Si eligen el metro, el tiempo de viaje será siempre igual a 30 minutos y el coste del viaje será de 1 unidad, mientras que si eligen el coche tanto el tiempo de viaje como el coste serán variables y dependerán de dos factores:

1. Factores casuales (clima, accidentes de tráfico, obras en las calles) cuyos efectos sobre el tiempo de viaje en coche serán elegidos al azar por el ordenador antes de cada período;

2. Congestión de tráfico, determinada por las elecciones de medio de transporte de todos los participantes.

El coste base del coche es igual a 1,5 unidades, pero si el tiempo real de viaje en coche es diferente del tiempo esperado de 25 minutos, tendrán una penalización de 0,5 unidades por cada cinco minutos de retraso o una ganancia de 0,5 unidades por cada 5 minutos ganados al tiempo de viaje previsto. 
El experimento comenzará cuando la siguiente ventana aparezca en la pantalla de su ordenador.

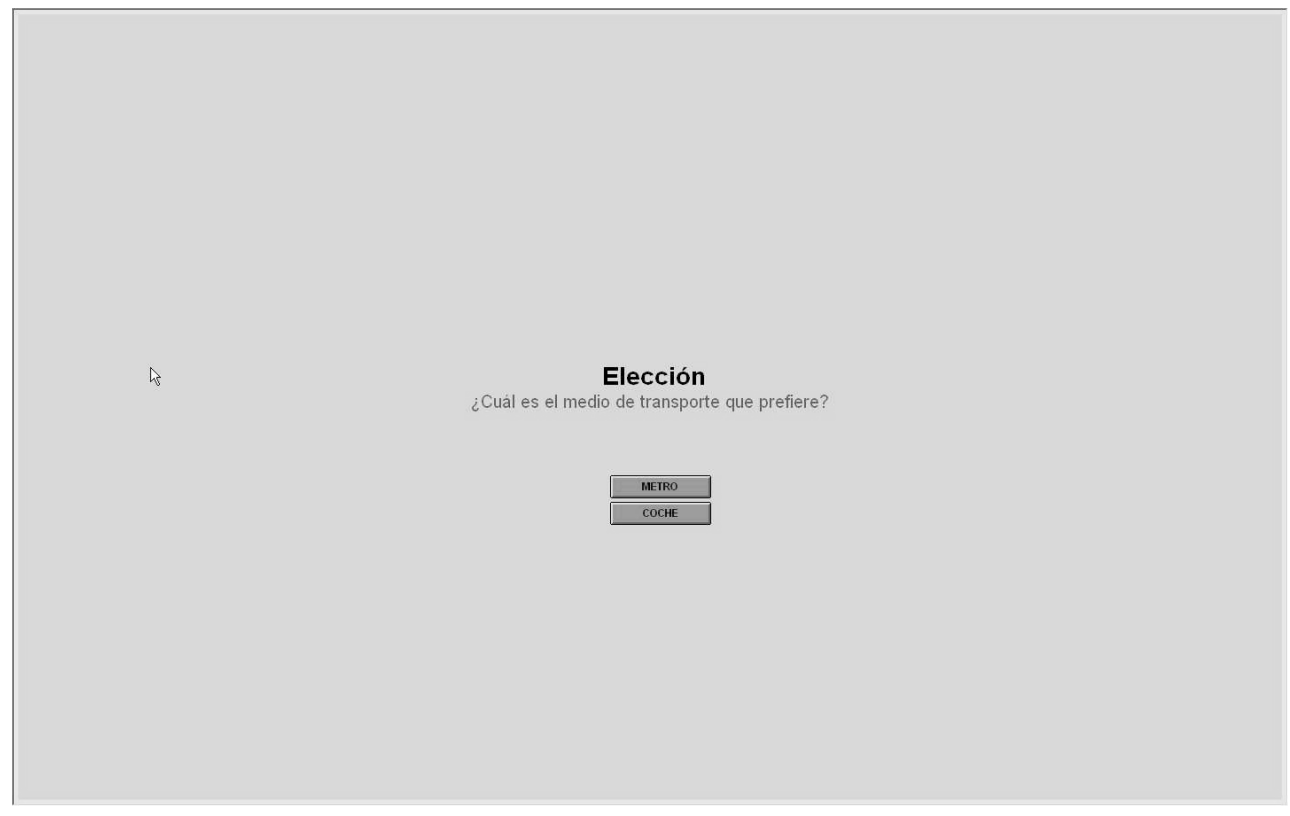

Usted debe elegir uno de los dos medios de transporte pulsando la opción adecuada. El tiempo disponible para tomar esta decisión se mostrará en la esquina superior izquierda de la ventana. Si el tiempo expira sin que se haya tomado una decisión, el ordenador seleccionará al azar el medio de transporte.

Después que todos los viajeros hayan tomado sus decisiones, si usted ha elegido el metro aparecerá la siguiente ventana en la pantalla de su ordenador (los números son ilustrativos). 


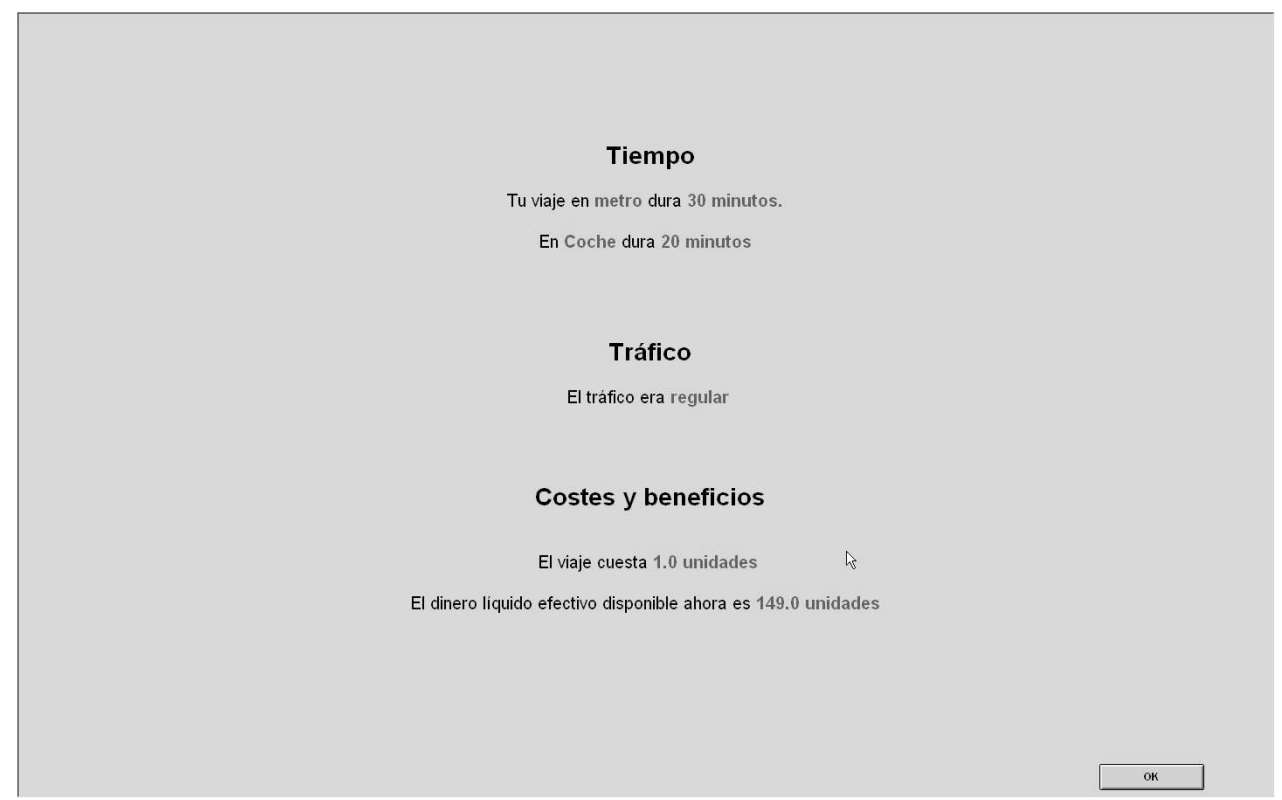

Si usted ha elegido el coche, les aparecerá la siguiente ventana en pantalla (los números son ilustrativos).

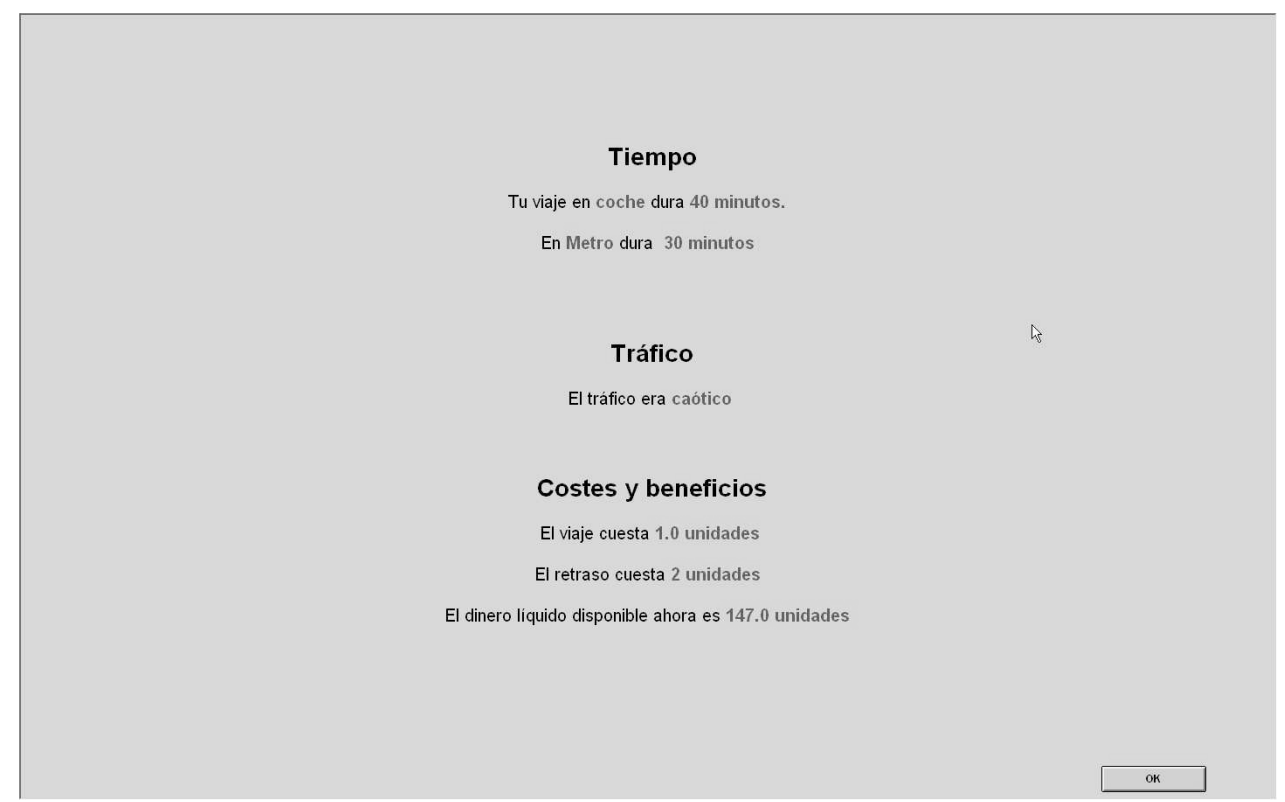


Como pueden ver, en la ventana encontrarán:

a) El tiempo real de viaje para los dos medios alternativos;

b) El nivel de congestión de tráfico definido como moderado, intenso o caótico, en relación al porcentaje de participantes que eligen el coche;

c) El coste monetario pagado por el viaje (incluyendo las penalizaciones o premios adicionales) y su número de unidades restantes.

Para pasar a la siguiente fase deberán pulsar el botón "OK" después de haber visto sus resultados.

Deberán repetir la misma decisión sobre los medios de transporte 50 veces. En cada fase, el procedimiento será el mismo. Para pagar todos los costes de viaje, cada participante recibirá al comienzo del experimento una dotación de 150 unidades. Su compensación final es la suma de las unidades restantes al final de las 50 fases. $\mathrm{Al}$ final del experimento sus beneficios totales serán traducidos en euros usando una tasa de conversión de 0,10 euros por cada unidad.

Por lo tanto, cuanto mayor sea el número de unidades que se tengan al final de los 50 períodos, mayor será el beneficio recibido.

Si necesitan información adicional es ahora el momento de preguntar. Una vez que haya comenzado el experimento no se puede parar para pedir información o aclaraciones.

Antes de que el experimento comience habrá sesiones de prueba para practicar sus decisiones. Cualquier beneficio o pérdida obtenida durante estas sesiones de prueba no se incluyen entre las ganancias totales del experimento.

¿Alguna pregunta?

Gracias por participar.

\section{Tratamiento autobús vs. Coche}

\section{BIENVENIDOS AL LABORATORIO}

Este es un experimento sobre toma de decisiones en economía. El IRPET (Instituto Regional para el Planeamiento Económico de la Toscana) y la Universidad de Siena han proporcionado los fondos para realizar esta investigación.

Cada uno de ustedes recibirá un premio de 5 euros por participar. Dependiendo de sus decisiones de inversión pueden obtener beneficios adicionales. Deberán seguir un conjunto de instrucciones para tomar las decisiones. El experimento es individual: tanto sus elecciones como sus ganancias serán confidenciales para todos excepto para ustedes mismos. El pago final se calcula automáticamente al final del experimento y se les pagará en efectivo al final de la sesión. Pueden comprobar su resultado con sus iniciales al final del experimento. 
Para garantizar los mejores resultados del experimento es necesario que respondan de forma individual, sin consultar a otros participantes.

Está permitido hacer preguntas después de que terminemos de repasar esta documentación con ustedes y/o justo antes de comenzar el experimento.

Habrá sesiones de prueba para mejorar su habilidad para tomar las decisiones antes de comenzar con el experimento real. Si están listos, sigan las instrucciones del experimento que aparecen más abajo.

\section{INSTRUCCIONES}

La decisión que cada uno de ustedes deberá tomar es elegir un medio de transporte. Están disponibles dos medios: autobús y coche. El tiempo de viaje tanto en autobús como en coche es incierto. El tiempo programado de viaje en autobús es de 32 minutos, mientras que el tiempo esperado de viaje en coche es de media 27 minutos. Después de tomar una decisión, se les informará del tiempo y coste reales de su viaje. Tanto el autobús como el coche tienen tiempos de viaje y costes que son variables y que dependen de dos factores:

1. Factores casuales (clima, accidentes de tráfico, obras en las calles) cuyos efectos sobre el tiempo de viaje en coche serán elegidos al azar por el ordenador antes de cada período;

2. Congestión de tráfico, determinada por las elecciones de modo de viaje de todos los participantes.

El coste base del autobús es igual a 1 unidad, pero si el tiempo real de viaje en autobús fuese diferente del tiempo esperado de 32 minutos tendrán una penalización de 0,5 unidades por cada cinco minutos de retraso con respecto al tiempo programado. Asimismo, el coste base del coche es igual a 1,5 unidades, pero si el tiempo real de viaje en coche fuese diferente del tiempo esperado de 27 minutos tendrán una penalización de 0,5 unidades por cada cinco minutos de retraso o una ganancia de 0,5 unidades por cada 5 minutos ganados al tiempo de viaje previsto. 
El experimento comenzará cuando la siguiente ventana aparezca en la pantalla de su ordenador.

\section{Elección}

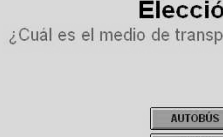


Usted debe elegir uno de los dos medios de transporte pulsando el botón adecuado. El tiempo disponible para tomar esta decisión se mostrará en la esquina superior izquierda de la ventana. Si el tiempo expira sin que se haya tomado una decisión, el ordenador seleccionará al azar el medio de transporte.

Después de que todos los viajeros hayan tomado sus decisiones, si ha elegido el autobús aparecerá la siguiente ventana en la pantalla de su ordenador (los números son ilustrativos)

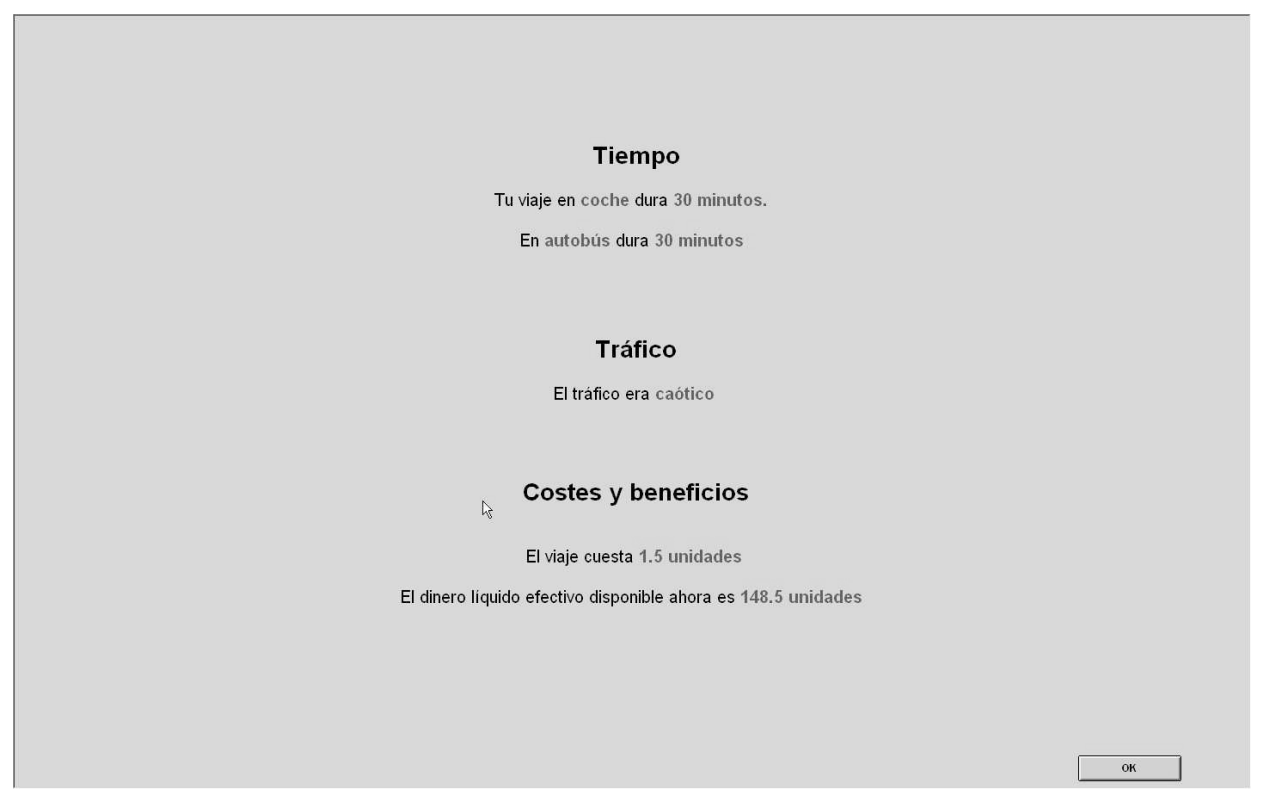


Si usted ha elegido el coche, les aparecerá la siguiente ventana en pantalla (los números son ilustrativos)

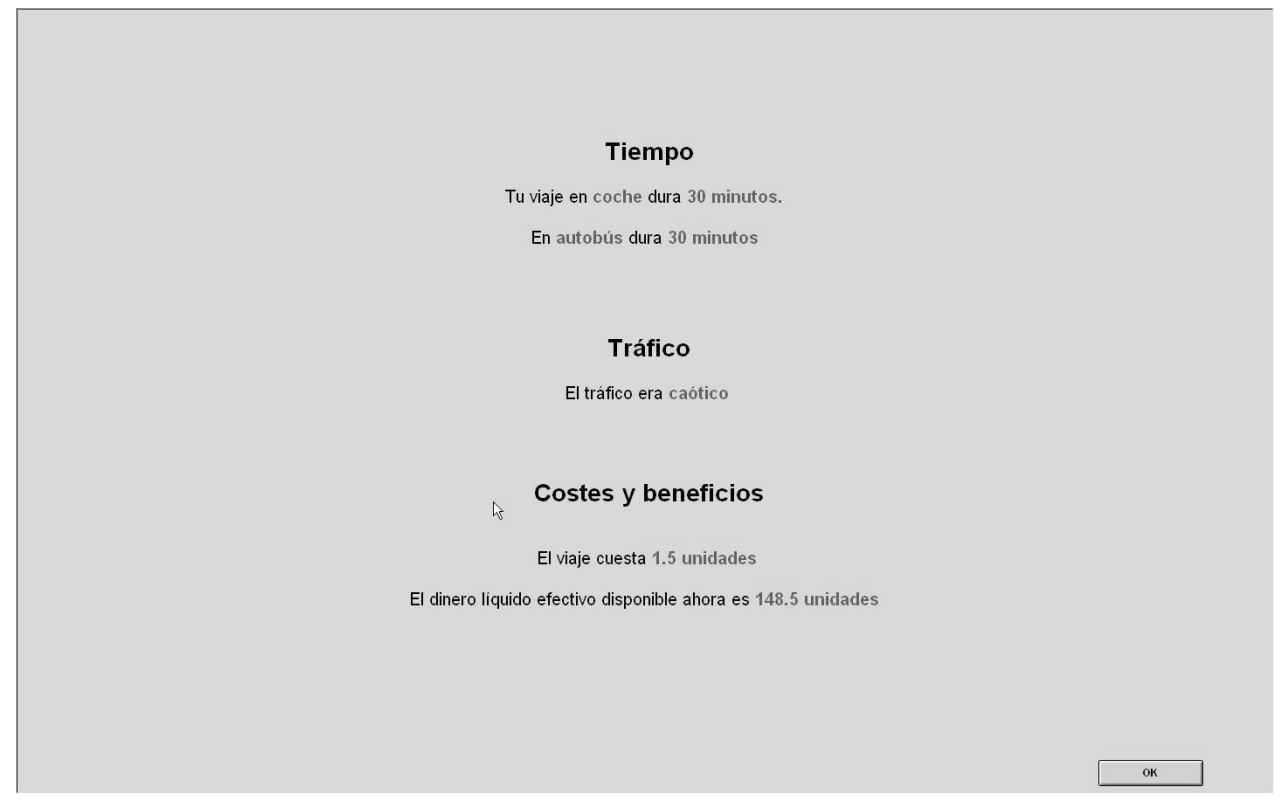

Como pueden ver, en la ventana encontrarán:

a) El tiempo real de viaje con los dos medios alternativos;

b) El nivel de congestión de tráfico definido como moderado, intenso o caótico, en relación al porcentaje de participantes que eligen el coche;

c) El coste monetario pagado por el viaje (incluyendo las penalizaciones o premios adicionales) y su número de unidades restantes.

Para pasar al siguiente período deberán pulsar el botón "OK" después de haber visto sus resultados.

Deberán repetir la misma decisión sobre los medios de transporte 50 veces. En cada período el procedimiento será el mismo. Para pagar todos los costes de viaje, cada participante recibirá al comienzo del experimento una dotación de 150 unidades. Su compensación final es la suma de las unidades restantes al final de los 50 períodos. Al final del experimento sus beneficios totales serán traducidos en euros usando una tasa de conversión de 0,10 euros por cada unidad.

Por lo tanto, cuanto mayor sea el número de unidades que se tengan al final de los 50 períodos, mayor será el beneficio recibido. 
Si necesitan información adicional es ahora el momento de preguntar. Una vez que haya comenzado el experimento no se puede detener para pedir información o aclaraciones.

Antes de que comience el experimento habrá sesiones de prueba para practicar sus decisiones. Cualquier beneficio o pérdida obtenida durante estas sesiones de prueba no se incluyen entre las ganancias totales del experimento.

¿Alguna pregunta?

Gracias por participar. 